\title{
Evaluation of the Universal Geocast Scheme For VANETs
}

\author{
Ben Bovée, Mohammad Nekoui, Hossein Pishro-Nik and Russell Tessier \\ Department of Electrical and Computer Engineering, University of Massachusetts, Amherst, MA
}

\begin{abstract}
Recently, a number of communications schemes have been proposed for Vehicular Ad hoc Networks (VANETs). A promising approach, the Universal Geocast Scheme (UGS), provides for a diverse variety of VANET-specific characteristics such as time-varying topology, protocol variation based on road congestion, and support for non line-of-sight communication. In this paper, the UGS protocol is extended to consider inter-vehicle multi-hop connections in intersections with surrounding obstructions. Since UGS is a probabilistic, repetition-based scheme, it supports the capacity-delay tradeoffs crucial for periodic safety message exchange. The approach is shown to support both vehicle-to-vehicle and vehicle-to-infrastructure communication. This research accurately evaluates this scheme using network (NS-2) and mobility (SUMO) simulators, verifying two crucial elements of successful VANETs, received packet ratio and message delay. A contemporary wireless radio propagation model is used to augment accuracy. Our results show a $6 \%$ improvement in received packet ratio combined with a decrease in average packet delay versus a previous, well-known intervehicle communication protocol.
\end{abstract}

Keywords-VANET, IEEE 802.11p, MAC

\section{INTRODUCTION}

An important aspect of the U.S. Department of Transportation Intelligent Transportation System's effort to advance transportation science is its Connected Vehicle initiative. The Connected Vehicle program advocates the use of dedicated short range communications (DSRC) to establish vehicle-to-vehicle (V2V) and vehicle-to-infrastructure (V2I) components for safety and congestion avoidance. The FCC has proposed the allocation of $75 \mathrm{MHz}$ in the $5.9 \mathrm{GHz}$ band specifically for communications between vehicles (V2V) and roadside equipment (RSE). Multiple channels within this band will be used for entertainment, safety, and control information. Connected Vehicle safety applications allow vehicles to have 360-degree awareness to inform a vehicle operator of hazards and non-visible situations. If the location, direction, and velocity of all vehicles in an immediate area are known, the safety system can inform a driver of potentially hazardous situations. The safety system can also help a driver in higher risk situations, such as highway lane changes, traffic merges when highway lanes decrease, intersections and many others.

Current Connected Vehicle safety messages fit into two categories: periodic and event-driven. Unlike less frequent event-driven messages [1], periodic status updates provide a 360-degree view to a driver. These periodic messages have varying size, broadcast range, and periods based on the type of safety information that is being transmitted in a given situation
[2]. Previous research used 200 byte packets [3] sent in a broadcast range of 80 meters [3] with a useful lifetime of 200 ms [4]. Generally, broadcasted inter-vehicle communications are targeted to a specific distance range, called the geocast range. The draft standard for the IEEE 802.11p DSRC protocol states that the fundamental access method for the medium access control (MAC) is carrier sense multiple access with collision avoidance (CSMA/CA) including the RTS/CTS and ACK handshake [5]. In the case of broadcast periodic safety messages, these handshakes represent a significant overhead, especially since RTS/CTS and ACK messages can be as large as the safety messages themselves. However, forgoing these messages gives rise to the hidden node problem. To combat this issue, multiple transmission repetitions for each message are used. Previous approaches [3] retransmit packets in randomly selected slots within a frame sequence. Generally, retransmission-based approaches for traffic intersections utilize direct single-hop communications between vehicles to minimize complexity. This simplicity can come at the cost of congestion. Multi-hop solutions, which involve intermediate vehicles or roadside equipment, have most often been deployed for open-road scenarios which exhibit limited transmission obstructions [6]. Most urban multi-hop protocols use eventdriven safety messages or use some form of the RTS/CTS and ACK handshaking [7] and focus on routing protocols [8].

Our new multi-hop inter-vehicle communications scheme implements periodic safety messages without the need for handshaking normally associated with routing protocols. The approach shows the effectiveness of multi-hop transmissions for periodic messages in dense urban intersections, which often are effected by line-of-sight obstructions. Individual node transmissions are scheduled using probabilistic factors based on distance to target and remaining packet lifespan. Both vehicles and roadside equipment located in the center of an intersection are used to retransmit packets. Transmission power is varied to reach targets within a defined geocast radius. The amount of time used to retransmit packets is probabilistically lengthened as packet retransmission count increases.

Our new Universal Geocast Scheme has been verified using several contemporary simulators. The core of our simulation environment is a significantly modified version of the NS-2 simulator [9]. The motion of vehicles approaching and traversing the intersection is modeled using SUMO [10], a vehicle mobility simulator. The accuracy of our simulations is enhanced with the use of a recently published radio propagation model [11], which is integrated into NS-2. Our new approach is experimentally contrasted against an existing inter-vehicle communication scheme from the Partners for 
Advanced Transit and Highways (PATH) group [3] using the enhanced NS-2 environment. A 6\% improvement in reception ratio and a substantial decrease in average delay are achieved for our new approach versus the previously-published approach.

\section{BACKGROUND}

The Universal Geocast Scheme (UGS) [1] is an 802.11compatible repetition-based MAC protocol, which fairly grants retransmission opportunities to contending neighbors. It differs from 802.11 in that there is a variable backoff window for retransmissions. The 802.11 protocol provides a random period of backoff time within a fixed contention window. The theoretical basis of UGS has been discussed previously [1], although this research presents the first experimental validation of the work using detailed simulation. Some additional protocol enhancements are also provided to the scheme.

In this paper, the UGS approach is contrasted with a leading previous inter-vehicle communication technique. The PATH team from the University of California, Berkeley [3] previously designed wireless local area networks to enable active vehicle safety systems. The work employs a repetition-based protocol with transmission acknowledgment called Asynchronous Fixed Repetition with Carrier Sensing (AFR-CS). The protocol randomly selects $k$ distinct time slots as potential transmission slots out of the $n$ slots constituting the lifetime of a packet. Prior to transmitting a packet, this protocol senses the availability of the transmission channel. Upon finding the channel idle, the packet is transmitted. If the channel is busy, the packet is dropped and transmission is deferred to the next selected time slot. The optimal number of retransmissions depends on the message rate, range, vehicular traffic density, and packet transmission time [3]. Unlike the PATH scheme, transmissions in our new protocol have more structure in how they are distributed over the lifetime of the packet. The new scheme has no set number of retransmissions. Instead, the amount of time between transmissions is altered. Unlike PATH, messages may be transmitted via either single or multihop broadcast depending on the current status of the vehicle.

To effectively simulate wireless communications, it is desirable to model the attenuation of the radio waves in their transmission environment. In an experiment described in Sal, et al. [11], a roadside antenna was deployed in an urban area in Tokyo with eight-story buildings on both sides of the street. A van equipped with a roof antenna received a $792.5 \mathrm{MHz}$ signal transmitted by the roadside antenna while in transit. The receiving signal strength and packet reachability (the number of successfully received packets divided by the number of transmitted packets) were collected and analyzed. Using processed data and existing radio propagation models it was possible to develop an intersection-based attenuation model, which is used in this research to better approximate real-world transmission conditions. One important aspect of this new Kangaku propagation model is that it has line of sight (LOS) and non-line of sight (NLOS) equations for calculating the propagation loss of a signal. The NLOS equations are based on the diffraction of the signal around the building, rather than the attenuation of the signals going through a building.

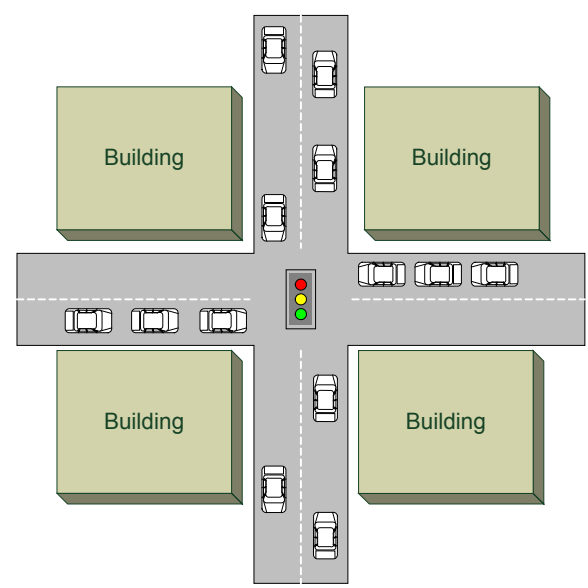

Figure 1. Intersection model for communication

\section{ENHANCED UNIVERSAL GEOCAST SCHEME}

For this paper, we consider UGS deployed under the scenario shown in Fig. 1. In addition to vehicles, the intersection contains a roadside equipment (RSE) unit located near the center of the intersection. Vehicles may be within lineof-sight (LOS) or not (NLOS) depending upon their placement in the intersection. Two identical lengths of road with two lanes each cross in the middle where a traffic light is located. Buildings are assumed to be located on all four corners of the intersection. Vehicles wait at the intersection stoplight until they are given a green light and continue straight across until they reach the other end of the road. Vehicles that are not in the intersection can transmit their own packets. Vehicles within the intersection can both transmit their own packets and retransmit packets received from other vehicles.

The highly dynamic topology of VANETs requires protocols that do not need a detailed description of the network topology in order to schedule packet transmissions. Our implemented protocol differentiates between the transmission behavior of vehicles in the intersection and outside the intersection (as determined by GPS). Since vehicles outside the intersection only transmit their own packets, their behavior will be described first. Following an initial transmission, a packet may be retransmitted at a random time between 0 and the backoff window. A distinctive feature of the UGS approach is a probabilistically-determined increase in the backoff time for each subsequent retransmission of the same packet. This random period of backoff time is normally determined from a contention window $(\mathrm{CW})$ as $\{0, \cdots, c w-1\}$. For this work, the backoff window is:

$$
b k=\left\{0, \cdots,\left\lfloor c w^{*} 2^{\left(i^{*} X+Y\right)}\right\rfloor-1\right\}
$$

where $i$ is the retransmission number of the packet, and $X$ and $Y$ are independent variables which present traffic density. In congested conditions, a packet which has already been transmitted will have to wait, on average, a longer time before retransmission. When many vehicles attempt transmissions, fewer transmissions are made per vehicle, limiting overcongestion. The fairness of this increasing backoff mechanism has been proved mathematically [4]. To correctly 


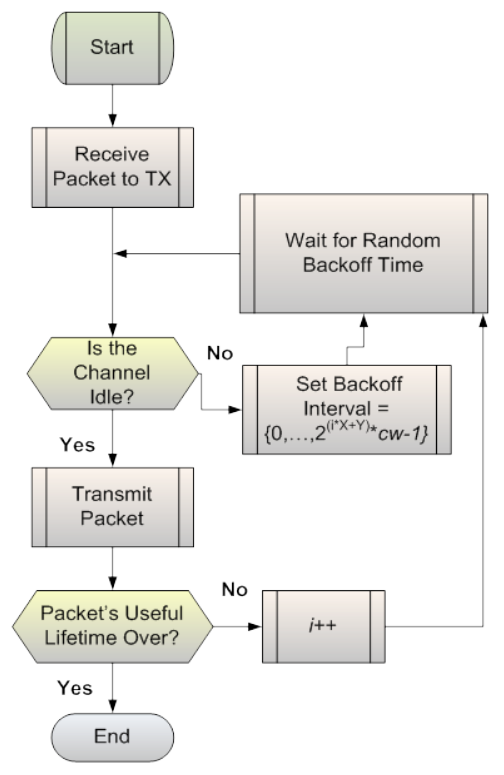

Figure 2. Algorithm for UGS MAC

set the backoff window, the number of times the packet has been retransmitted has to be known. The packet header can be easily updated to track its retransmission number. The retransmission number is incremented in the MAC before the packet is passed to the PHY layer. The receiving function of the MAC sets the retransmission to 0 . A summary of the steps used for packet retransmission is shown in Fig. 2.

Vehicles located within an intersection differ from ones outside the intersection in that they can forward packets from other vehicles as well as transmit their own. Each node retransmission uses a single or multiple hops based on the geometry of the intersection surroundings and the remaining useful lifetime of the packet. Since intersection-bound vehicles can transmit both their own packets and packets originating from other vehicles, dynamic decisions must be made to determine which packet is forwarded at which time. As shown in Fig. 3, packets generated by the vehicle that are targeted for retransmission are stored in the Own Queue $(O Q)$, while packets from other vehicles are stored in the Forwarding Queue $(F Q)$. Packets stored in $F Q$ are ordered using two criteria. Packets that have been transmitted the fewest times are placed at the front of the queue. These packets are then ordered by their remaining useful lifespan. Packet headers contain a time stamp, which helps determine their remaining lifespan. The remaining packets in the queue are then ordered in a similar fashion (first as a group by transmission count and then by remaining lifespan within the group).

The selection of a packet from either $O Q$ or $F Q$ is based on the remaining lifespan of the packet at the top of the $F Q$. This $F Q$ packet is transmitted with a probably $t$, which is proportional to its lifespan. The packet at the top of the $O Q$ is transmitted with probability l-t. After a packet is selected, it is transmitted using set criteria. Retransmissions of packets originating from intersection-bound vehicles are transmitted using (1), in a similar fashion to vehicles located outside the intersection. However, retransmissions of packets which originate at other vehicles are confined to a fixed backoff window to maximize their chance of reception.

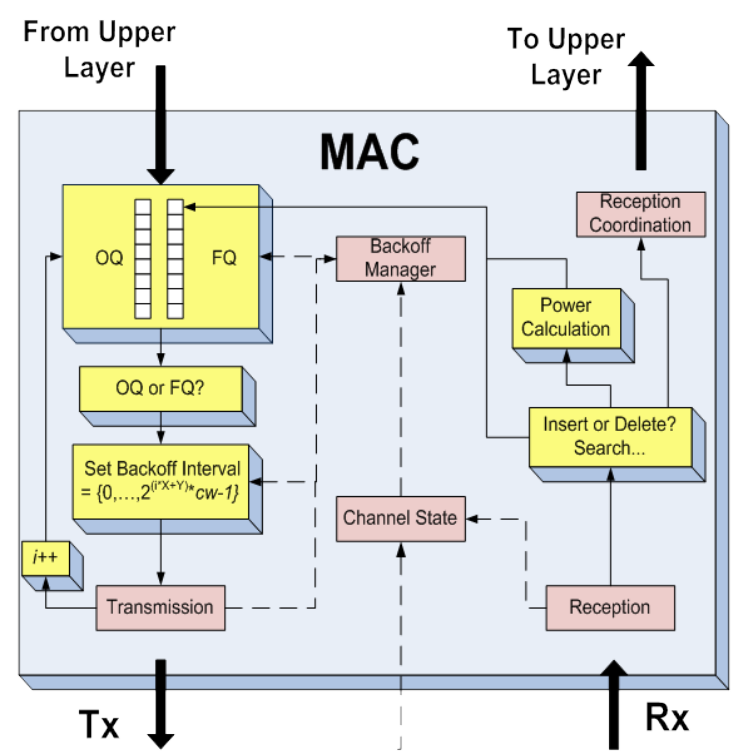

Figure 3. Architecture of UGS MAC

This value was experimentally determined to be 1920 time slots. Packets with expired lifespans are automatically ejected from associated queues. Fig. 3 illustrates several of these features. Carrier sensing is represented by the channel state box while the insert or delete box represents decisions made during packet reception.

Several optimizations are considered for intersection-bound vehicles. If a vehicle detects the transmission of packet in its $F Q$ by another vehicle, the packet is removed. This detection indicates the close proximity of another vehicle already forwarding the message. Additionally, for multi-hop transmissions, if the transmission target for an intermediate hop is within a closer proximity than the original vehicle packet source, transmission power can be reduced accordingly. Power adjustment is aided by the inclusion of the physical location of the packet source in the packet header. This operation is represented by the power calculation box in Fig. 3 .

\section{Simulation Set-Up}

The core of our protocol evaluation was performed using a heavily-modified version of NS-2, a network simulator [9]. This open source discrete event simulator is targeted at networking research with a focus on network protocols. Our version of NS-2 is based on recent additions that support IEEE 802.11 implementations of medium access control (MAC) and physical (PHY) layers [12].

An urban intersection scenario was constructed in NS-2 to test both UGS and PATH schemes. Vehicles are located within LOS or NLOS depending upon their placement in the intersection and the Kangaku radio propagation model is integrated into the simulator accordingly. Fig. 4 shows how a packet sent at a power level to overcome NLOS attenuation at a range of 72.8 meters will produce a much longer LOS broadcast resulting in unwanted interference. Traffic flows were accurately modeled using the "Simulation of Urban MObility" (SUMO) tool, which accounts for vehicles traveling 


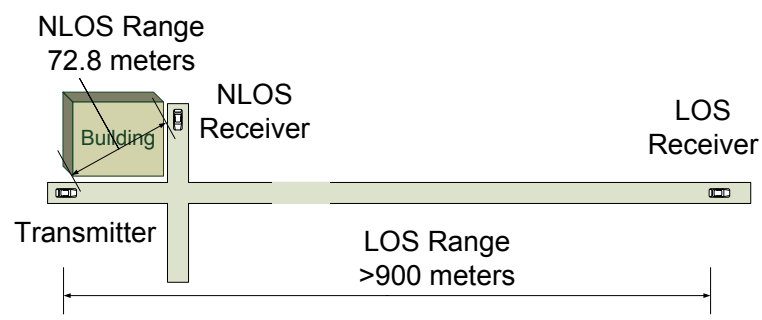

Figure 4. NLOS vs. LOS range of a transmission

at various speeds. SUMO is an open source, highly portable, microscopic road traffic simulation package designed to handle large road networks [10].

The intersection model shown in Fig. 1 was used for simulations. Road length and vehicle density were varied from simulation to simulation. Each vehicle produces an original message every $200 \mathrm{~ms}$, which is subsequently retransmitted based on the backoff strategies described in Section III. The broadcast range of the messages is 80 meters at a frequency of $5.18 \mathrm{GHz}$ and the packets are 200 bytes in size with a useful lifetime of $200 \mathrm{~ms}$. For single hop transmissions, the power is calculated for each transmission such that every vehicle within an 80-meter radius of the transmitting vehicle will receive the broadcast. Only vehicles within the 80 -meter broadcast range of the sender count towards calculated results. For each individual simulation, the reception ratio, average number of transmissions per packet, and average delay per packet were recorded. The reception ratio is the number of vehicles within the original 80 -meter broadcast range that actually receive the transmission divided by the numbers of vehicles that were targeted. The average packet transmissions (APT) value is an average of the number of times each packet is actually broadcast. The average delay per packet is the average amount of time it takes each packet to be received by vehicles within the $80 \mathrm{~m}$ broadcast range. The simulations were run for a total of 20.4 seconds each. Results were calculated for the middle 20 seconds. Results obtained from vehicles in the $100 \mathrm{~m}$ of each road furthest from the intersection were ignored to account for edge effects. The TraceExporter extension was used to translate the SUMO mobility traces into an NS-2 compatible format.

Several protocol specific modifications were made to NS-2 to support both the modified UGS protocol and the PATH protocol used for comparison. A parameter was added to allow for a variable number of PATH retransmission attempts. For UGS, the $X$ and $Y$ parameters noted in (1) were made userdefinable. A global variable was set up so that $X$ and $Y$ values could be input via a Tcl file on a per-simulation basis. This input variable is bound to the variable in the $\mathrm{C}++$ file used in determining the size of the backoff interval. Power calculation functions were also formulated in NS-2 to calculate the power so that packets would not be broadcast outside their original broadcast range. Additionally, the Kangaku radio propagation model, described in Section II, was implemented.

\section{RESULTS}

Intersection scenarios utilizing 500, 800, 900, and 1,200 meter roads on all four sides of an intersection were evaluated in four separate experiments. As shown in Fig. 5, as the length

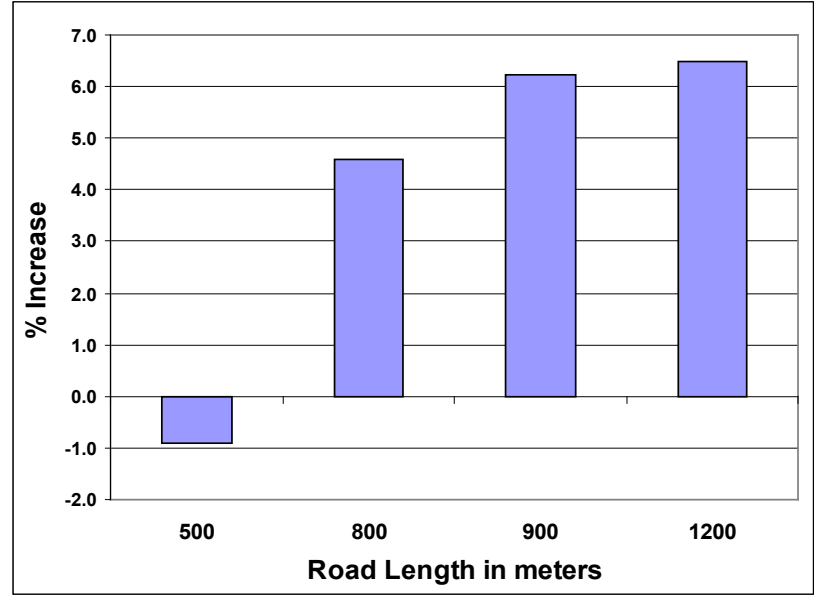

Figure 5. Reception ratio increase of UGS vs. PATH for different road lengths

of the roads increased, the reception ratio of UGS versus PATH improved. UGS has a decrease in performance by $1 \%$ for 500 meter roads, but an increase of $4.6 \%, 6.2 \%$, and $6.5 \%$ for 800 , 900 , and 1200 meter roads, respectively. Results shown are for UGS without the use of RSE. The longer roads allow for improved interference modeling of single-hop transmissions. The added road length increases inter-vehicle transmission interference, especially when NLOS vehicles within the 80 meter broadcast range are considered.

An important factor in the performance of both PATH and UGS schemes is vehicle density. The PATH paper [3] indicated that the maximum flow density for highway simulations is an inter-vehicle spacing of roughly 30 meters. For the 900-meter road intersection scenario, the average number of vehicles evaluated during 20 -second simulations is 337 , leading to an average inter-vehicle spacing of 23.7 meters. Taking this maximum density into account, simulations were repeated considering traffic densities of $75 \%, 50 \%$, and $25 \%$ of the maximum. As Table I demonstrates, as vehicle density decreases, the advantage of the UGS scheme is reduced. The last two columns represent the performance increase of UGS with and without the use of an RSE located in the center of an intersection to forward packets. In the densest simulations, UGS has an increased performance over the PATH scheme by over $6 \%$.

As noted in Section III, the backoff window size is an important metric in packet retransmission. A sweep was performed to evaluate the best possible values for the $X$ and $Y$ values used in (1). Fig. 6 indicates that an $X$ value of roughly 9 is ideal for increased reception ratio when used with a $Y=8$

TABLE I. Intersection Reception Ratio

\begin{tabular}{|c|c|c|c|c|c|}
\hline \multirow{2}{*}{$\begin{array}{c}\text { Max } \\
\text { Flow } \\
\% \\
\end{array}$} & \multirow[t]{2}{*}{ PATH } & \multirow[t]{2}{*}{ UGS } & \multirow{2}{*}{$\begin{array}{l}\text { UGS } \\
\text { with } \\
\text { RSE }\end{array}$} & \multicolumn{2}{|c|}{ UGS \% Improvement } \\
\hline & & & & $\begin{array}{c}\text { Without } \\
\text { RSE }\end{array}$ & $\begin{array}{l}\text { With } \\
\text { RSE }\end{array}$ \\
\hline 100 & 0.839 & 0.891 & 0.892 & 6.2 & 6.3 \\
\hline 75 & 0.881 & 0.919 & 0.920 & 4.4 & 4.4 \\
\hline 50 & 0.948 & 0.963 & 0.960 & 1.5 & 1.2 \\
\hline 25 & 0.983 & 0.981 & 0.984 & -0.2 & 0.2 \\
\hline
\end{tabular}




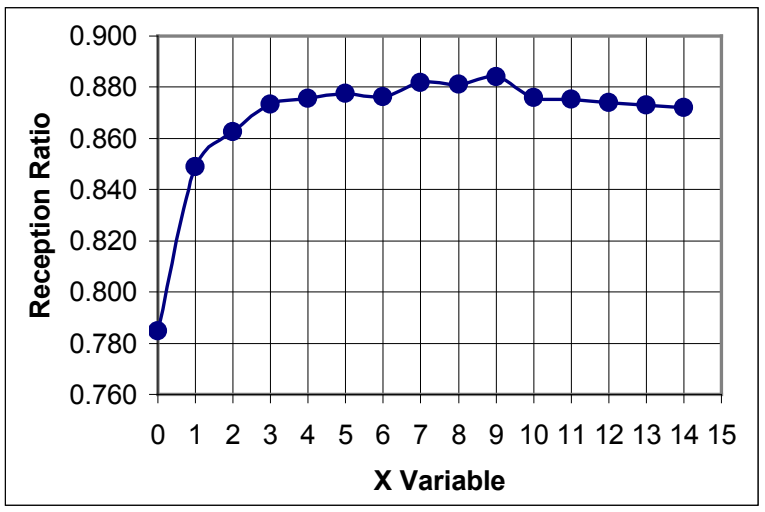

Figure 6. Reception ration vs. X for UGS experiment with $900 \mathrm{~m}$ roads

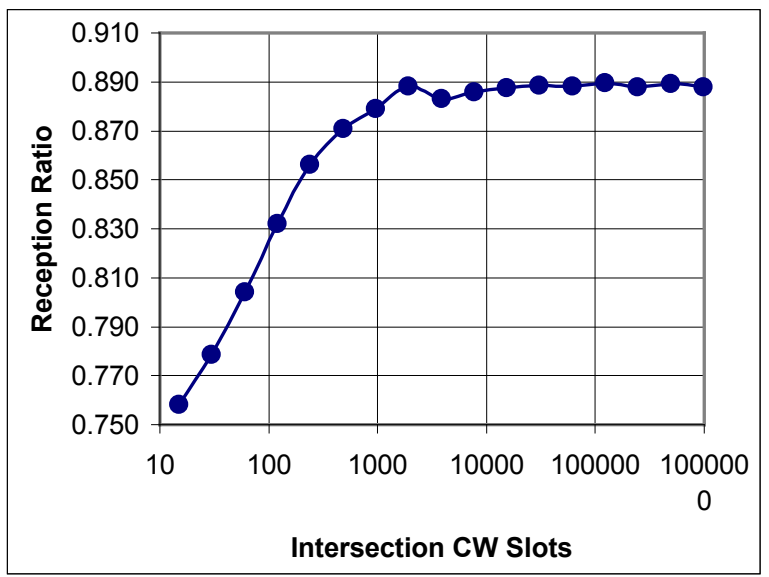

Figure 7. Effect of intersection $\mathrm{CW}$ on reception ratio

value. A similar experiment was performed to determine the appropriate value for $Y$. It is important that the $Y$ variable not be too large to avoid overly lengthening the backoff window. If the backoff window is longer than the lifetime of the packet, the packet may not be resent. However, if $X$ or $Y$ is too small, excessive packet retransmissions may occur, causing congestion. Another important variable that impacts UGS is the set $C W$ for intersection node forwarding, especially when an RSE is used. Fig. 7 indicates the reception ratio of UGS in a 900-meter road length intersection scenario under varying intersection $C W$ values.

The second important measure of performance is delay. The average packet delay for PATH and UGS over the four different vehicle densities can be seen in Table II. The table demonstrates the decrease in delay of the UGS.

TABLE II. Average packet delay of UGS vs. Path

\begin{tabular}{|c|c|r|}
\hline $\begin{array}{c}\text { Max Flow } \\
\mathbf{\%}\end{array}$ & $\begin{array}{c}\text { PATH } \\
\text { in } \mathbf{~ m s}\end{array}$ & $\begin{array}{c}\text { UGS } \\
\text { in } \mathbf{~ m s}\end{array}$ \\
\hline $\mathbf{1 0 0}$ & 76.28 & 13.01 \\
\hline $\mathbf{7 5}$ & 74.47 & 12.74 \\
\hline $\mathbf{5 0}$ & 63.42 & 8.33 \\
\hline $\mathbf{2 5}$ & 46.62 & 5.37 \\
\hline
\end{tabular}

In the maximum flow simulations, UGS, on average, only took $20 \%$ of the time that the PATH scheme did in successfully transmitting packets to intended receivers. The large difference in average delay of the two protocols can be attributed to the PATH protocol picking random times to transmit, regardless of the channel state. UGS will transmit immediately upon finding the channel idle. Only as the number of transmissions increases does the backoff, and thus wait time, increase.

\section{CONCLUSIONS}

This research describes a detailed evaluation of a new multi-hop inter-vehicle communication protocol, which has been optimized for obstructed intersections. This modified Universal Geocast Scheme performs $6 \%$ better with decreased delay than an accepted, previously-published approach [3] for periodic inter-vehicle messages which do not use RTS/CTS and ACK messages. The UGS repetition-based scheme allows for both LOS and NLOS packet transfer. Our results have been generated using a modified NS-2 simulator, a recentlydeveloped radio propagation model, and a traffic mobility simulator.

\section{REFERENCES}

[1] M. Nekoui, H. Pishro-Nik, "A Universal Geocast Scheme for Vehicular Ad Hoc Networks," Proc. IEEE Consumer Communications and Networking Conference, Jan. 2010, pp. 1-5.

[2] R. Chen, W. Jin, and A. Regan, "Broadcasting safety information in vehicular networks: issues and approaches" IEEE Network: The Magazine of Global Internetworking, Vol. 24, No. 1, Jan.-Feb. 2010, pp. 20-25.

[3] Q. Xu, T. Mak, J. Ko, R. Sengupta, "Medium Access Control Protocol Design for Vehicle-Vehicle Safety Messages" IEEE Transactions on Vehicular Technology, Vol. 56, Nr. 2, March 2007, pp. 499-518.

[4] M. Nekoui, H. Pishro-Nik, "Reliable inter-vehicle communications for vehicular ad hoc networks" Proc. International Wireless Internet Conference, Nov. 2008, pp. 84.

[5] IEEE Std 802.11 $\mathrm{p}^{\mathrm{TM}}$-2010 Standard for Information technology - Part 11: Wireless LAN Medium Access Control (MAC) and Physical Layer (PHY) Specifications

[6] J. Mittag, F. Thomas, J. Harri, and H. Hartenstein, "A comparison of single- and multi-hop beaconing in VANETs", Proc. ACM International Workshop on VehiculAr InterNETworking", Sept. 2009, pp. 69-78

[7] G. Korkmaz, E. Ekici, and F. Ozguner, "Black-burst-based multihop broadcast protocols for vehicular networks," IEEE Transactions on Vehicular Technology, vol. 56, no. 5, Sept. 2007, pp. 3159 - 3167.

[8] Z. Mo, H. Zhu, K. Makki, and N. Pissinou, "MURU: A Multi-Hop Routing Protocol for Urban Vehicular Ad Hoc Networks." Proc. International Conference on Mobile and Ubiquitous Systems, Jul. 2006, pp.1-8.

[9] S. Ivanov, A. Herms, and G. Lukas, "Experimental validation of the NS2 wireless model using simulation, emulation, and real network," Proc. Workshop on Mobile Ad-Hoc Networks, Mar. 2007, pp. 433-444.

[10] Simulation of Urban Mobility (SUMO), Institute of Transportation Systems at the German Aerospace Center http://sourceforge.net/apps/mediawiki/sumo/index.php?title=Main_Page Accessed Dec. 2010

[11] S. Sal, et al., "Field evaluation of UHF radio propagation for an ITS safety system in a urban environment," IEEE Communications Magazine, Nov. 2009, pp. 120-127

[12] Q. Chen, F. Schmidt-Eisenlohr, D. Jiang, M. Torrent-Moreno, L. Delgrossi, H. Hartenstein, "Overhaul of IEEE 802.11 modeling and simulation in ns-2", Proc. ACM Symposium on Modeling, Analysis, and Simulation of Wireless and Mobile Systems, Oct. 2007, pp. 159-168. 